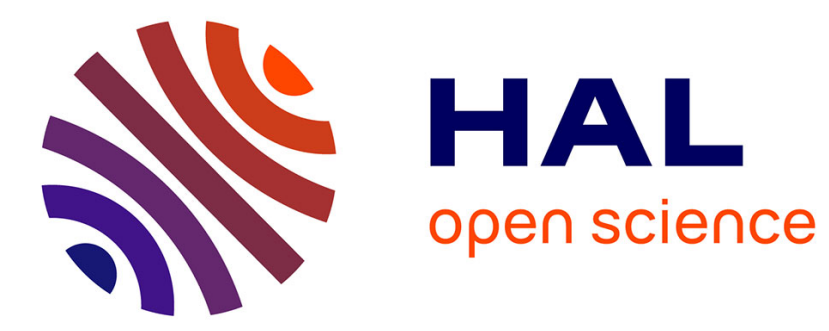

\title{
Topological Shaping of Light by Closed-Path Nanoslits
}

Etienne Brasselet, Gediminas Gervinskas, Gediminas Seniutinas, Saulius

\author{
Juodkazis
}

\section{To cite this version:}

Etienne Brasselet, Gediminas Gervinskas, Gediminas Seniutinas, Saulius Juodkazis. Topological Shaping of Light by Closed-Path Nanoslits. Physical Review Letters, 2013, 111 (19), pp.193901. 10.1103/PhysRevLett.111.193901 . hal-00880432

\section{HAL Id: hal-00880432 \\ https://hal.science/hal-00880432}

Submitted on 1 Mar 2018

HAL is a multi-disciplinary open access archive for the deposit and dissemination of scientific research documents, whether they are published or not. The documents may come from teaching and research institutions in France or abroad, or from public or private research centers.
L'archive ouverte pluridisciplinaire HAL, est destinée au dépôt et à la diffusion de documents scientifiques de niveau recherche, publiés ou non, émanant des établissements d'enseignement et de recherche français ou étrangers, des laboratoires publics ou privés.

\section{(ㄷ)(1) $\$$}

Distributed under a Creative Commons Attribution - NonCommerciall 4.0 International 


\title{
Topological Shaping of Light by Closed-Path Nanoslits
}

\author{
Etienne Brasselet* \\ Université Bordeaux, CNRS, Laboratoire Ondes et Matière d'Aquitaine, UMR 5798, F-33400 Talence, France \\ Gediminas Gervinskas, Gediminas Seniutinas, and Saulius Juodkazis \\ Centre for Micro-Photonics, Faculty of Engineering and Industrial Sciences, Swinburne University of Technology, \\ Hawthorn, Victoria 3122, Australia \\ Melbourne Centre for Nanofabrication, Australian National Fabrication Facility, Clayton, Victoria 3168, Australia
}

(Received 20 March 2013; published 5 November 2013)

\begin{abstract}
We propose a discrete set of continuous deformation of a circular nanoslit to generate and control optical vortices at the microscopic scale. The process relies on the interplay between the spin and orbital angular momentum degrees of freedom of light mediated by appropriate closed-path nanoslits milled on a thin gold film. Topological shaping of light is experimentally demonstrated in the visible domain. Moreover, all experimental observations are quantitatively validated by a simple model that takes into account the transverse manipulation of the optical phase via the space-variant form birefringence of subwavelength slits.
\end{abstract}

DOI: 10.1103/PhysRevLett.111.193901

PACS numbers: 42.25.-p, 42.50.Tx, 42.81.Gs

The orbital angular momentum (OAM) carried by a light beam is associated with its phase spatial distribution. Some features of the OAM confer to it unique benefits relative to the spin angular momentum, which is connected to the polarization state of light. A striking property is the formally unlimited discretization of the OAM per photon, as is the case for Laguerre-Gaussian beams [1] that represent a complete basis with infinite dimension made of optical vortex beams. This promises many applications, as discussed in recent reviews [2,3] or anticipated from recent findings in optical communications [4]. In particular, further development relies on the ability to realize optical vortex generators at small scale.

In practice, several attempts to reduce the size of optical vortex generators were undertaken by straightforward downsizing of existing demonstrations at the centimeter scale. For instance, $5 \mu \mathrm{m}$ diameter spiral phase plates have been produced by direct laser writing [5]. The miniaturization of so-called Pancharatnam-Berry phase macroscopic optical elements $[6,7]$ is another example. This has been done by using natural [8] or field-induced [9-11] liquid crystal topological defects that allow realizing tunable optical vortex generators at the 1-100 $\mu \mathrm{m}$ length scale. Other approaches have also been developed, such as the realization of $\sim 10 \mu \mathrm{m}$ diameter integrated vortex beam emitters [12] and plasmonics options relying either on achiral [13] or chiral [14,15] nanostructures. However, the route to "singular nanophotonics" - the controlled generation of optical singularities at the nanoscale-still remains challenging.

Here, we propose to use curved closed-path nanoslits (width-to-wavelength ratio $<1$ ) homeomorphic to the circle to achieve topological shaping of light at the microscale. The concept basically relies on the introduction of cusped design. Interestingly, cusped slits with width-to-wavelength ratio $\sim 10^{3}$ have been recently noticed for their intriguing scalar diffraction properties [16].

Noting that a straight nanoslit behaves as a form birefringent retarder whose main axes are perpendicular and parallel to it [17], a simple understanding to our geometrical approach can be inferred from Jones formalism. To this aim, let us consider a singly connected closed path $\mathcal{C}$ that defines a curvilinear birefringent optical element characterized by a constant birefringent phase retardation $\Delta$, as shown in Fig. 1(a). Neglecting diffraction, the expression of the output light field along $\mathcal{C}$ for a normally incident circularly polarized plane wave with Jones vector $\mathbf{E}_{\text {in }}=$ $E_{0} \mathbf{c}_{\sigma}$, where $\mathbf{c}_{\sigma}=(\mathbf{x}+i \sigma \mathbf{y}) / \sqrt{2}, \sigma= \pm 1$, refers to the circular polarization basis, is $\mathbf{E}_{\text {out }}(\phi)=E_{0}\left[\cos (\Delta / 2) \mathbf{c}_{\sigma}+\right.$ $\left.i \sin (\Delta / 2) e^{i 2 \sigma \beta(\phi)} \mathbf{c}_{-\sigma}\right]$, where $\beta(\phi)$ is the local tangent angle to $\mathcal{C}$. Consequently, by designing the contour $\mathcal{C}$ such as the circulation of $\beta$ around the closed path satisfies $s=(1 / 2 \pi) \oint_{\mathcal{C}} d \beta$, with $s$ a half-integer, one obtains an azimuthal phase dependence $\Phi(\phi)=2 \sigma \beta(\phi)$ for the $\mathbf{c}_{-\sigma}$
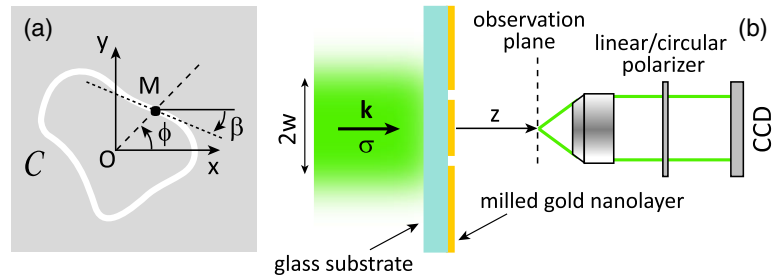

FIG. 1 (color online). (a) Geometry and definition of characteristic angles $\beta$ and $\phi$ for a closed-path nanoslit circuit $\mathcal{C}$ homeomorphic to the circle. (b) Illustration of the experimental setup. A circularly polarized collimated Gaussian beam with wave vector $\mathbf{k}$, waist diameter $2 w$, and helicity $\sigma= \pm 1 \mathrm{imp}-$ inges at normal incidence on the nanoslit plane. 
(a)
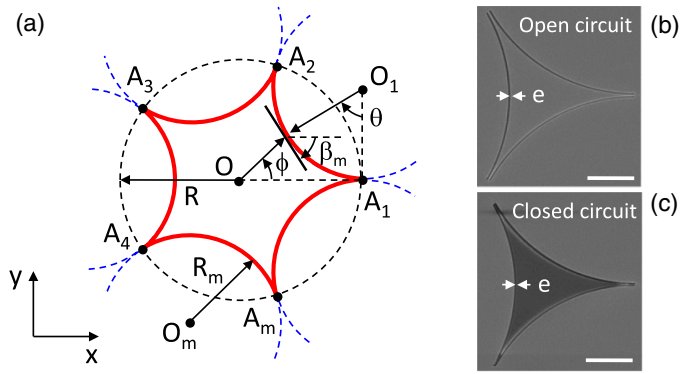

FIG. 2 (color online). (a) Closed-path topological nanoslit of order $m$, here with $m=5$ (thick curve), where $R$ is the radius of the circle in which is inscribed the structure and $R_{m}$ is the radius of the circles that generate the design. (b),(c) Scanning electron microscope (SEM) image of the case $m=3$ in its open and closed forms, with $R=10 \mu \mathrm{m}$ and slit width $e=150-200 \mathrm{~nm}$. The scale bar is $5 \mu \mathrm{m}$. The contrast difference of the two SEM images is due to different electrical charging effects between the two kinds of structures.

polarized output field just after the device that satisfies $\ell=(1 / 2 \pi) \oint_{\mathcal{C}} d \Phi$, with $\ell$ integer. That is to say, an optical field component associated with a topological charge $\ell$ is obtained.

The particular case when $\mathcal{C}$ is a circle, which leads to $\ell= \pm 2$, has been reported only recently [13]. Here, we unveil the generalization to arbitrary values of $\ell$ by mere continuous deformation of the circle. The main idea is to introduce a contour $\mathcal{C}$ with multiple cusps, for instance, with a design consisting of $m$ arcs of circles $(m \geq 3)$ with radius $R_{m}=R \tan (\pi / m)$, where $R$ is the radius in which the structure is inscribed; see Fig. 2. In this case, one gets $\ell=\sigma(2-m)$. This can be grasped from the variation of the tangent angle $\beta$ to the structure along one of the $m$ arcs of the circle, namely, $\Delta \beta=\beta(\phi=2 \pi / m)-\beta(\phi=0)=$ $2 \pi / m-\pi$ [see Fig. 2(a)], which leads by definition to a topological charge that is $m$ times $2 \sigma \Delta \beta$ divided by $2 \pi$.

In practice, two kinds of topological nanoslits have been fabricated, namely, the "open" and the "closed" forms, as shown for $m=3$ in Figs. 2(b) and 2(c), respectively. The samples were made on $500 \mu \mathrm{m}$ thick borosilicate glass substrates. The substrates were first cleaned by washing them in an ultrasonic bath for $5 \mathrm{~min}$ in deionized water, acetone, and isopropanol. Layers of titanium and gold (6 and $60 \mathrm{~nm}$, respectively) were magnetron sputtered (AXXIS, Kurt J. Lesker) on the cleaned substrates, and vortex generators were made by direct Ga-ion beam lithography (IonLiNE, Raith). A $300 \mathrm{pA}$ current was used to mill 150-200 nm wide grooves throughout the gold and titanium layers.

Closed loop topological nanoslits of order $m=3$ to 8 are shown in the upper part of Fig. 3, for $R=10 \mu \mathrm{m}$. Ensuing polarization conversion is observed at $\lambda=532 \mathrm{~nm}$ wavelength under normal incidence illumination by a Gaussian laser beam with $2 w=280 \mu \mathrm{m}$ diameter and circular polarization state [Fig. 1(b)]. The structure is

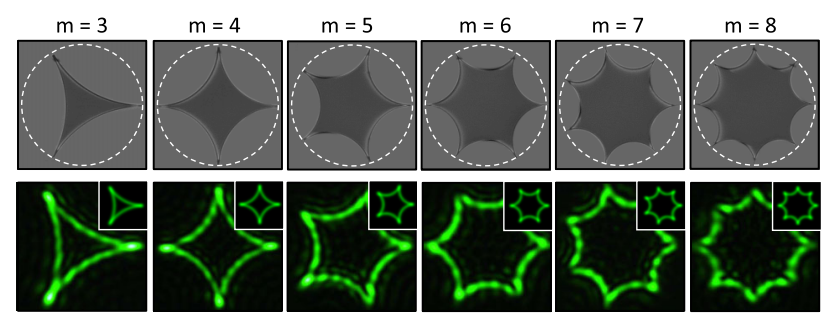

FIG. 3 (color online). Upper row: SEM images of closed loop nanoslits of order $m=3$ to 8 with $R=10 \mu \mathrm{m}$ (see dashed circle) and slit width $e=150-200 \mathrm{~nm}$. Bottom row: Optical imaging of the slit at $532 \mathrm{~nm}$ wavelength under crossed circular polarizers. Insets: Calculated convoluted nanoslit images accounting for the numerical aperture of the imaging system.

then imaged by a microscope objective by selecting the $\mathbf{c}_{-\sigma}$ polarized component of the output field; see the bottom part of Fig. 3. Note that the observed nonuniform intensity patterns are explained by the finite numerical aperture $(\mathrm{NA}=0.25)$ of the microscope objective and illustrated in the insets of Fig. 3, which display the calculated intensity distribution of an evenly illuminated structure convoluted by a Gaussian function with waist radius $0.61 \lambda / \mathrm{NA}$. Hot spots are indeed expected at the tip of each cusp.

Quantitatively, the polarization conversion efficiency $\eta$ is measured as the ratio between the power carried by the $\mathbf{c}_{-\sigma}$ polarized component of the light field transmitted by the nanoslit and the total transmitted power by the nanoslit. We find $\eta=0.7 \% \pm 0.1 \%$, whatever the open or closed nature of the structure and its radius, as shown in Fig. 4. Such a modest value merely emphasizes the use of nonoptimized structures rather than intrinsic limitations of the polarization conversion process. Larger values of efficiency can indeed be achieved by appropriate nanoslit width and height for a given wavelength and material [17]. For instance, almost 50\% efficiency has been reported in Ref. [13] for $e / \lambda \simeq 0.2$ at $\lambda=830 \mathrm{~nm}$ and a $200 \mathrm{~nm}$ thick gold layer. In contrast, here we have $e / \lambda \simeq 0.3-0.4$ at $\lambda=532 \mathrm{~nm}$ and a more than 3 times thinner gold layer.

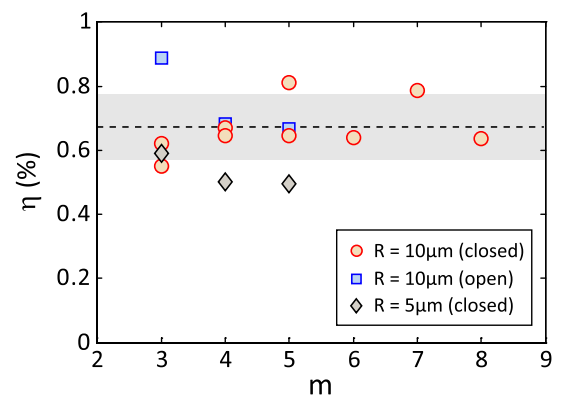

FIG. 4 (color online). Polarization conversion efficiency $\eta$ of nanoslit structures of different order $(m)$, size $(R)$, and nature (open or closed forms). The dashed line is the mean value, and the gray area refers to the standard deviation range. 


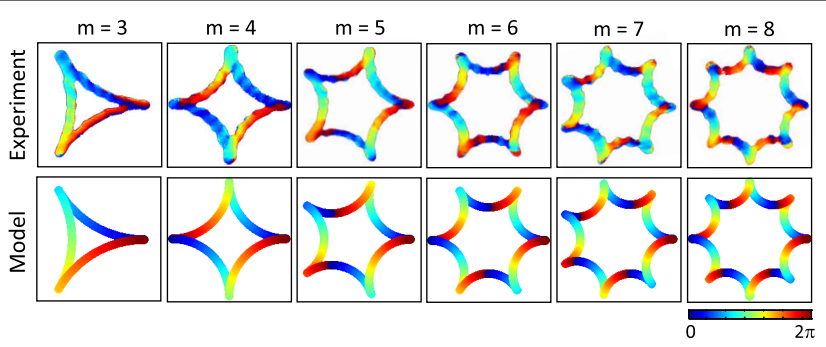

FIG. 5 (color online). Map of the phase distribution of the generated optical vortices for nanoslits of order $m=3$ to 8 , in the plane of the nanoslit, with $\sigma=-1$. Upper row: Experimental data with $R=10 \mu \mathrm{m}$. The shown data refer to the location where intensity is measured for the contracircularly polarized field component; see Fig. 3. Bottom row: Model.

In addition, we notice that $\eta$ is not only dictated by a birefringent phase delay condition but also involves dichroism [18]. Here, the relatively low thickness of the gold layer probably plays a crucial role on the value of $\eta$. We have checked that finite-difference time-domain simulations give indeed efficiency of the order of a percent with present parameters. The topological shaping of light, however, is basically dictated by the order $m$ of the structure.

The azimuthal behavior of the phase of the $\mathbf{c}_{-\sigma}$ polarized light field component at the nanoslit output can be retrieved experimentally. This is done from spatially resolved polarimetric analysis of the output beam in order to determine the map of the polarization ellipse azimuth angle, which equals half the latter phase [8]. The results are displayed in the upper row of Fig. 5 and compared with the theoretical behavior shown in the bottom row of Fig. 5, which qualitatively agree with the data, whatever is $m$. In fact, the agreement is quantitatively validated as well. This is demonstrated in Fig. 6, where $\Phi_{m}(\phi)$ data are shown for $m=3,4$, and 5 and compared with the piecewise expression generated from the first arc $A_{1} A_{2}$ by introducing the angle $\theta$ [see Fig. 2(a)], namely, $\Phi_{m}(\phi)=-2 \sigma \theta(\phi)$, with the following relationship between $\phi$ and $\theta: \tan \phi=R_{m}(1-\cos \theta) /\left(R-R_{m} \sin \theta\right)$.

As presented above, topological shaping of light is achieved in the plane of the nanoslit. The question of
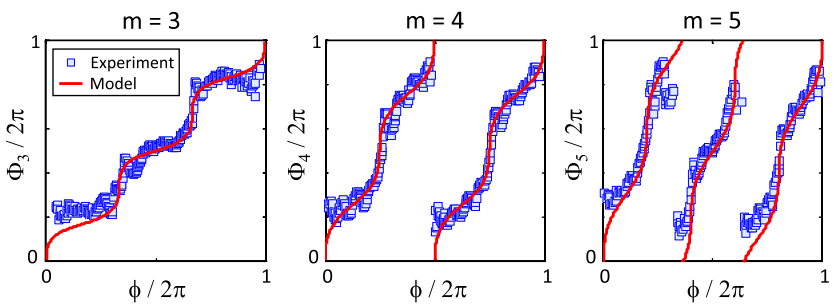

FIG. 6 (color online). Nonuniform azimuthal phase profile $\Phi_{m}(\phi)$ of the generated optical vortices for nanoslits of order $m=3$, 4, and 5 with $R=10 \mu \mathrm{m}$ and $\sigma=-1$. Markers: Experimental data averaged over three different structures (one in open form and two in closed form). Curve: Model. how it manifests in the far field is investigated in what follows. This is done by observing the transverse intensity distribution vs the propagation distance $z$. The case $m=3$ is shown in Fig. 7 for a nanoslit with $R=5 \mu \mathrm{m}$ and $\sigma=-1$. In this figure, the intensity distribution of the $\mathbf{c}_{-\sigma}$ polarized output light field component exhibits a one-arm spiral pattern that blooms with propagation, hence revealing the generation of on-axis optical phase singularity with unit topological charge, as expected. Generalization to higher-order nanoslits is summarized in Fig. 8 for $m=3$ to 8 , hence $\ell=1$ to 6 , where the upper part of the figure displays the observed $\ell$-arm spiraling patterns. Noticeably, no additional beam has been used, as is quantitatively supported next.

For this purpose, the light field at the output of the circular polarizer $E_{\text {out }}$ is calculated from the Fresnel diffraction integral in the paraxial approximation by taking into account (i) the residual transmission of the gold film (we measure $3 \%$ power transmission, hence an amplitude transmission $t_{\text {film }}=0.17$ ), (ii) the finite contrast of the circular polarizer made of a zero-order quarter-wave plate placed at $45^{\circ}$ from a linear polarizer (we measure a residual $0.05 \%$ transmittance for the "blocked" polarization state, hence $t_{\sigma} \sim 0.02$, whereas we have $t_{-\sigma} \sim 0.9$ for the "passing-through" polarization state), and (iii) the finite polarization conversion efficiency $\eta$. We thus get

$$
\begin{aligned}
E_{\text {out }}(\rho, \vartheta, z) \propto & -\frac{i}{\lambda z} \iint\left\{t_{\text {film }} t_{\sigma} \overline{\mathcal{A}}(r, \phi)+\left[\sqrt{1-\eta} t_{\sigma}\right.\right. \\
& \left.\left.+\sqrt{\eta} t_{-\sigma} e^{i \Phi_{m}(\phi)}\right] \mathcal{A}(r, \phi)\right\} \\
& \times G(r) e^{i \pi / \lambda z\left[r^{2}+\rho^{2}-2 r \rho \cos (\phi-\vartheta)\right]} r d r d \phi,
\end{aligned}
$$

where $G(r)=\exp \left(-r^{2} / w^{2}\right), \mathcal{A}(r, \phi)$ refers to the area defined by the nanoslit aperture (equals 1 where the gold layer has been milled, 0 otherwise), and $\overline{\mathcal{A}}(r, \phi)$ is the complementary screen. In addition, $r=\sqrt{x^{2}+y^{2}}$ is the distance from the $z$ axis and $\phi$ the azimuthal angle in the nanoslit plane, whereas $\rho$ and $\vartheta$ are the polar coordinates in the observation plane located at the distance $z$ from the plane of the aperture. Taking into account that $w \gg R$ and that observations are performed at $z$ values much smaller than the incident Gaussian beam Rayleigh distance $z_{0} \sim 10 \mathrm{~cm}$, Eq. (1) can be recast in the form

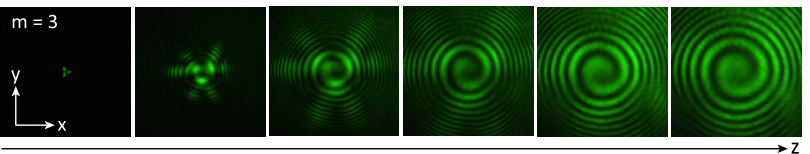

FIG. 7 (color online). Propagation of the contracircularly polarized optical field component for the fundamental nanoslit structure $m=3$ with $R=5 \mu \mathrm{m}$. The first image refers to the nanoslit plane $(z=0)$, and others, from left to right, correspond to $d z=20 R$ propagating steps. All images are on the same scale, and acquisition time increases with $z$. 


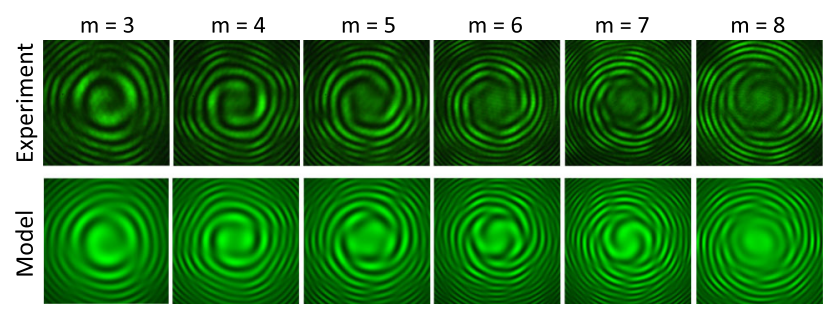

FIG. 8 (color online). Characteristic single beam self-spiraling optical textures for nanoslits of order $m=3,4,5,6,7$, and 8 . The number of spiral arms is $m-2$, which corresponds to the topological charge of the optical vortex generated by the closed loop nanoslit. Upper row: Experimental data with $R=10 \mu \mathrm{m}$. Bottom row: Model.

$$
\begin{aligned}
E_{\text {out }}(\rho, \vartheta, z) \propto & t_{\text {film }} t_{\sigma} e^{-\rho^{2} / w^{2}}-\frac{i}{\lambda z} \iint_{\mathcal{A}}\left[\left(\sqrt{1-\eta}-t_{\text {film }}\right) t_{\sigma}\right. \\
& \left.+\sqrt{\eta} t_{-\sigma} e^{i \Phi_{m}(\phi)}\right] \\
& \times e^{i \pi / \lambda z\left[r^{2}+\rho^{2}-2 r \rho \cos (\phi-\vartheta)\right]} r d r d \phi
\end{aligned}
$$

The resulting intensity patterns $I(\rho, \vartheta, z)=\left|E_{\text {out }}(\rho, \vartheta, z)\right|^{2}$ are displayed in the bottom row of Fig. 8. Fair agreement with experimental data is found. Note that both $t_{\text {film }} \neq 0$ and $t_{\sigma} \neq 0$ are necessary to provide the intensity pattern with spiraling features.

Also, the wave front handedness of the contracircularly polarized field generated by a nanoslit can be switched, say, from right to left, by changing the handedness of the incident circular polarization state, whatever is the value of $m$. This is illustrated in Fig. 9 in the cases of $m=3$ and $m=4$. Such a behavior is analog to the polarization control of the wave front helicity reported in the case for macroscopic optical elements with space-variant birefringent phase retardation [19].

Although spiraling patterns with $\ell$ arms are observed off axis, the data suggest that far-field topological shaping of light is not associated with the appearance of higher-order optical vortices with topological charge $\ell$ as $m$ increases. Instead, as confirmed by simulations at finite values of propagating distance $z$, we observed a constellation of off-axis unit charge vortices; see Fig. 8. In fact, this is a general feature of high-order phase singularities [20,21]. In the present case, it is the broken axial symmetry of the geometry that is basically at the origin of the vortex

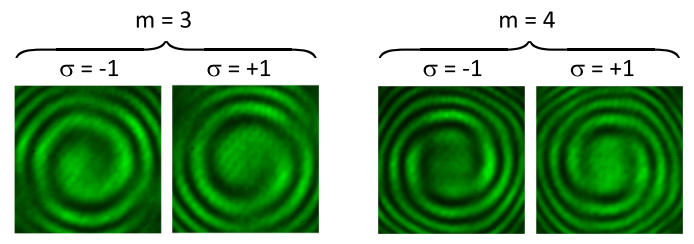

FIG. 9 (color online). Polarization switching of the topological charge for nanoslits of order $m=3$ and 4 with $R=10 \mu \mathrm{m}$, where $\sigma= \pm 1$ refers to the helicity of the incident beam on the structures. splitting. This is emphasized in the upper and middle rows of Fig. 10, where the scalar Fraunhofer diffraction intensity and phase patterns of the output $\mathbf{c}_{-\sigma}$ polarized field produced by nanoslits of order $m=3$ to 8 sitting on an ideally opaque milled film are shown. Calculations are done, up to an unimportant multiplying real factor that quantifies the polarization conversion efficiency, following the expression

$$
E_{-\sigma}(\mu, \nu) \propto \iint_{\mathcal{C}} e^{i 2 \sigma \beta(u, v)} e^{-i 2 \pi(R / \lambda)(\mu u+\nu v)} d u d v,
$$

where $\exp (i 2 \sigma \beta)$ is the complex transmittance along the nanoslit for the $\mathbf{c}_{-\sigma}$ polarized output field, with $u=x / R$ and $v=y / R$, whereas $\mu$ and $\nu$ are the reduced reciprocal coordinates, which vary between -1 and 1 . This confirms a far-field on-axis topological shaping for $m=3$ and 4 , whereas vortex splitting is more pronounced as $m$ increases. Still, the circulation of the phase along the circle that passes by intensity maxima equals $2 \pi \ell$ with a monotonic and quasilinear azimuthal phase dependence, as shown in the bottom row of Fig. 10.

Combining topological diversity with ultrafast polarization driven OAM modulation abilities, the realization of ultrathin plasmonic vortex nanoplates operating on arbitrary OAM subspaces could be envisioned. Our findings might also lead to novel designs of plasmonic OAM couplers based on spin-controlled transmission [22] towards the elaboration of miniaturized OAM sorters with enhanced functionalities. Efficient OAM sorting down to the single photon level is indeed restricted so far to macroscopic refractive devices [23], whereas microscale sorters are only in their infancy [24]. Being based on geometrical considerations, our approach could be formally downsized, as emphasized in Ref. [25] in the axially symmetric case. Since the nanofabrication state of the art allows
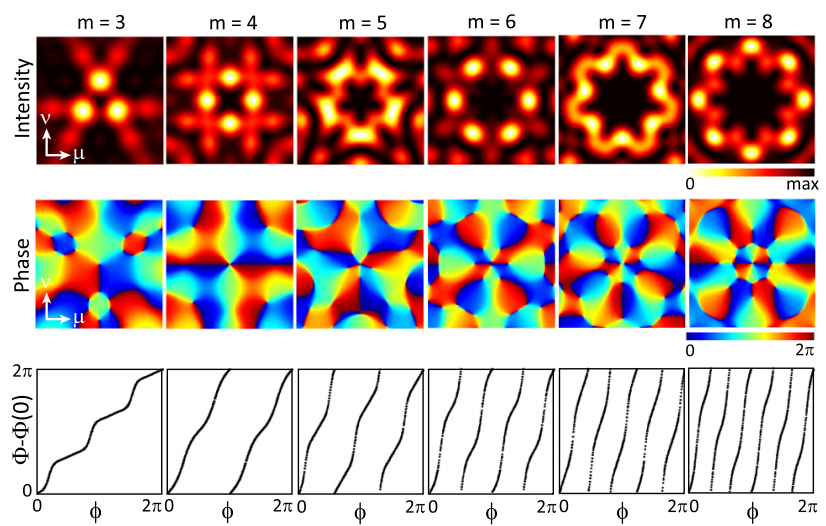

FIG. 10 (color online). Far-field characteristics for $m=3,4$, 5, 6, 7, and 8. First and second rows: Fraunhofer diffraction intensity and phase patterns in the reciprocal coordinate system, here displayed in the range $-0.1 \leq(\mu, \nu) \leq 0.1$. Third row: Azimuthal dependence of the phase along the circle that passes by the intensity maxima. Simulation parameters: $\sigma=-1$, $w / R=0.02, \lambda=500 \mathrm{~nm}$, and $R=10 \mu \mathrm{m}$. 
considering 10-20 nm width nanoslits [26], individual nanostructures with radius down to $100 \mathrm{~nm}$ become possible to fabricate. This promises further development of singular nanophotonics. However, downsizing in the closed form case is expected to alter the sharpness of the cusp, hence the optical performances of the structure. Finally, we note that one could obtain fractional values of $\ell$ by an appropriate modification of the proposed geometry.

We are grateful to Lorenzo Rosa who provided us with numerical simulations regarding the expected value of the polarization conversion efficiency.

*e.brasselet@loma.u-bordeaux1.fr

[1] L. Allen, M. W. Beijersbergen, R. J.C. Spreeuw, and J. P. Woerdman, Phys. Rev. A 45, 8185 (1992).

[2] A. M. Yao and M. J. Padgett, Adv. Opt. Photonics 3, 161 (2011).

[3] M. Padgett and R. Bowman, Nat. Photonics 5, 343 (2011).

[4] J. Wang, J.-Y. Yang, I. M. Fazal, N. Ahmed, Y. Yan, H. Huang, Y. Ren, Y. Yue, S. Dolinar, M. Tur, and A. E. Willner, Nat. Photonics 6, 488 (2012).

[5] E. Brasselet, M. Malinauskas, A. Žukauskas, and S. Juodkazis, Appl. Phys. Lett. 97, 211108 (2010).

[6] G. Biener, A. Niv, V. Kleiner, and E. Hasman, Opt. Lett. 27, 1875 (2002).

[7] L. Marrucci, C. Manzo, and D. Paparo, Appl. Phys. Lett. 88, 221102 (2006).

[8] E. Brasselet, N. Murazawa, H. Misawa, and S. Juodkazis, Phys. Rev. Lett. 103, 103903 (2009).

[9] E. Brasselet and C. Loussert, Opt. Lett. 36, 719 (2011).

[10] E. Brasselet, Phys. Rev. Lett. 108, 087801 (2012).

[11] R. Barboza, U. Bortolozzo, G. Assanto, E. Vidal-Henriquez, M. G. Clerc, and S. Residori, Phys. Rev. Lett. 109, 143901 (2012).
[12] X. Cai, J. Wang, M. J. Strain, B. Johnson-Morris, J. Zhu, M. Sorel, J.L. OBrien, M.G. Thompson, and S. Yu, Science 338, 363 (2012).

[13] P. F. Chimento, P. F. A. Alkemade, G. W. 't Hooft, and E. R. Eliel, Opt. Lett. 37, 4946 (2012).

[14] P. Zilio, E. Mari, G. Parisi, F. Tamburini, and F. Romanato, Opt. Lett. 37, 3234 (2012).

[15] Y. Gorodetski, A. Drezet, C. Genet, and T. W. Ebbesen, Phys. Rev. Lett. 110, 203906 (2013).

[16] G. Martinez-Niconoff, J. Munoz-Lopez, J. Silva-Barranco, A. Carbajal-Dominguez, and P. Martinez-Vara, Opt. Lett. 37, 2121 (2012).

[17] P.F. Chimento, N. V. Kuzmin, J. Bosman, P.F. A. Alkemade, G. W. 't Hooft, and E. R. Eliel, Opt. Express 19, 24219 (2011).

[18] E. Brasselet, Opt. Lett. 38, 3890 (2013); P. F. Chimento, P. F. A. Alkemade, G. W. 't Hooft, and E. R. Eliel, Opt. Lett. 38, 3891 (2013).

[19] L. Marrucci, C. Manzo, and D. Paparo, Phys. Rev. Lett. 96, 163905 (2006).

[20] M. S. Soskin, V. N. Gorshkov, M. V. Vasnetsov, J. T. Malos, and N.R. Heckenberg, Phys. Rev. A 56, 4064 (1997).

[21] V.G. Denisenko, A. Minovich, A. S. Desyatnikov, W. Krolikowski, M. S. Soskin, and Y. S. Kivshar, Opt. Lett. 33, 89 (2008).

[22] Y. Gorodetski, N. Shitrit, I. Bretner, V. Kleiner, and E. Hasman, Nano Lett. 9, 3016 (2009).

[23] M. P. J. Lavery, D. J. Robertson, G. C. G. Berkhout, G. D. Love, M. J. Padgett, and J. Courtial, Opt. Express 20, 2110 (2012).

[24] P. Genevet, J. Lin, M. A. Kats, and F. Capasso, Nat. Commun. 3, 1278 (2012).

[25] O. G. Rodríguez-Herrera, D. Lara, K. Y. Bliokh, E. A. Ostrovskaya, and C. Dainty, Phys. Rev. Lett. 104, 253601 (2010).

[26] G. Gervinskas, G. Seniutinas, L. Rosa, and S. Juodkazis, Adv. Opt. Mater. 1, 456 (2013). 